\title{
Depressão e suicídio em idosos no Brasil: revisão de literatura
}

\author{
Depression and suicide in the elderly in Brazil: literature review
}

Depresión y suicidio en ancianos en Brasil: revisión de literatura

Milena Alessandra Oliveira da Costa ${ }^{1 *}$, Ana Carolina de Moraes Cruz¹.

\section{RESUMO}

Objetivo: Descrever com base na literatura, quais evidências científicas publicadas nos últimos 10 anos relatam sobre a ideação suicida em idosos que possuem como principal fator de risco a depressão. Métodos: Trata-se de um estudo realizado por meio do método de revisão integrativa da literatura com abordagem qualitativa, através de buscas em bases de dados de publicações online. Foram selecionados nove artigos, cujo critério de inclusão foram artigos científicos que contemplassem a temática no período de 2010 a 2019. Resultados: Observou-se que os principais fatores de risco relacionados ao suicido em idosos é a depressão, ocasionada pela aposentadoria, problemas de relacionamento familiar, perda de habilidades, diminuição de possibilidade de escolhas etc. Além disso, mulheres idosas são as que mais pensam no ato de suicido, no entanto, é o gênero masculino que mais pratica. Considerações finais: Identificar e compreender os sintomas depressivos em idosos aumenta a eficácia de medidas de prevenção para a ideação suicida nesse grupo.

Palavras-chave: Envelhecimento, Sintomas depressivos, Suicídio.

\section{ABSTRACT}

Objective: Describe, based on the literature, what scientific evidence published in the last 10 years reports on suicidal ideation in the elderly who have depression as their main risk factor. Methods: This is a study carried out using the integrative literature review method with a qualitative approach, through searches in databases of online publications. Nine articles were selected, whose inclusion criteria were scientific articles covering the theme from 2010 to 2019. Results: It was observed that the main risk factors related to suicide in the elderly is depression, caused by retirement, family relationship problems, loss of skills, decreased choice etc. In addition, elderly women are those who think most about the act of suicide, however, they are the male gender who practices the most. Final considerations: Identifying and understanding depressive symptoms in the elderly increases the effectiveness of preventive measures for suicidal ideation in this group.

Key words: Aging, Depressive symptoms, Suicide.

\section{RESUMEN}

Objetivo: Describa, con base en la literatura, qué evidencia científica publicada en los últimos 10 años informa sobre la ideación suicida en los ancianos que tienen la depresión como su principal factor de riesgo. Métodos: Este es un estudio realizado utilizando el método integrador de revisión de literatura con un enfoque cualitativo, a través de búsquedas en bases de datos de publicaciones en línea. Se seleccionaron nueve artículos, cuyos criterios de inclusión fueron artículos científicos que cubrieron el tema de 2010 a 2019. Resultados: Se observó que los principales factores de riesgo relacionados con el suicidio en los ancianos son la depresión, causada por la jubilación, problemas de relación familiar, pérdida de habilidades, disminución de la elección etc. Además, las mujeres mayores son las que más piensan en el acto de suicidio, sin embargo, son el género masculino que más práctica. Consideraciones finales: Identificar y comprender los síntomas depresivos en los ancianos aumenta la efectividad de las medidas preventivas para la ideación suicida en este grupo.

Palabras clave: Envejecimiento, Síntomas depresivos, Suicidio.

1Universidade Nilton Lins (UNINILTONLINS), Manaus - AM. *E-mail: maalessandra10@gmail.com 


\section{INTRODUÇÃO}

O envelhecimento é um acontecimento que acontece em todas as partes do mundo que a cada dia vem tornando-se mais expressivo e gerador de pesquisas nos países desenvolvidos e subdesenvolvidos (BATISTA AS, et al., 2008). Em âmbito nacional, como é o caso do Brasil, o crescimento exponencial da população da terceira idade resulta da combinação de variáveis transição demográficas com as intensas mudanças sociais e culturais ocorridas, que concomitantemente configuram-se como causa e consequência (CRUZ DT, et al., 2010).

Devido ao setor da saúde estar despreparada para atender a demanda de uma população com idade superior aos 60 anos de idade, as mudanças na pirâmide etária brasileira não têm sido adequadamente acompanhadas pelo poder público e áreas da saúde, em função de um excesso de doenças crônicodegenerativas que levam a limitações funcionais e cognitivas (SAMPAIO LS, et al., 2009; VALCARENGHI RV, 2011).

Sintomas depressivos caracterizam-se como um distúrbio de um resultado de uma combinação de fatores da área afetiva ou do humor, que desempenha intenso impacto funcional e envolve diversos aspectos de ordem biológica, psicológica e social, tendo como principais sintomas a falta de humor e a perda de interesse em atividades cotidianas (CARREIRA L, et al., 2011). Para Barreto $\mathrm{J}$ (2006), as perdas que o idoso vai experimentando, a nível pessoal e social, criam situações de luto cuja resolução pronta nem sempre é fácil, sobretudo se certos fatores negativos se fazem sentir.

Alguns estudos indicam que a depressão é uma doença subdiagnosticada e subtratada, comum e recorrente em indivíduos da terceira idade (MEDEIROS JML, 2010). As mudanças ocorridas no contexto social e cultural como aposentadoria, impossibilidade de exercer a profissão, viuvez, residir em asilos, acarretam uma espécie de "morte social". E é a partir da vivência singular que cada idoso tem diante dessas adversidades da vida que o mesmo experimenta sentimentos negativos, que por sua vez, desencadeiam em isolamento social, solidão, angústia, cujo desfecho pode ser, a ideação suicida ou a tentativa de suicídio (TEIXEIRA LMF, 2010).

No Brasil, as mudanças demográficas ocorreram de forma tão acelerada que a população idosa em 2011 alcançou 23,5 milhões, o que estava previsto para ser alcançado apenas em 2020 (BRASIL, 2014). Com o aumento da longevidade, corroborando com os altos índices de suicídio de idosos, a prevenção do suicídio nessa faixa etária torna-se um desafio para os setores sociais e de saúde (CAVALCANTE FG e MINAYO MCS, 2012). A análise espaço-temporal da mortalidade por suicídio de idosos realizada no período de 2000 a 2014 indica que houve um crescimento significativo: no período de 2000 a 2014 com 19.806 óbitos por suicídio de idosos no Brasil (SANTOS EGO, et al., 2017).

É notório que o suicídio na terceira idade provocado por fatores de depressão constitui hoje um dos graves problemas para as sociedades das mais diversas partes do mundo. Minayo MCS e Cavalcanti FG (2010) em seu estudo, afirmam que os dados de autodestruição em pessoas maiores de 60 anos são muito elevados, em que a razão entre tentativas e suicídios consumados é muito próxima, quase 2:1. Esses dados evidenciam a importância de levar em consideração os mínimos sinais de um ato suicida em pessoas desta idade.

Estudos sobre essa temática, principalmente a nível nacional, ainda são escassos e necessitam de ampliação dos pontos de análise dessa discussão científica e social. Considerando que o profissional de Enfermagem possui em um dos seus eixos a ação educativa, é importante compreender a ocorrência do impacto social e os serviços de saúde oferecidos para a população acima dos 60 anos de idade. Sendo assim, esta pesquisa teve como objetivo efetuar uma revisão da literatura, a fim de obter uma compreensão acerca de estudos sobre envelhecimento, depressão e suicídio.

\section{MÉTODOS}

O estudo refere-se a uma revisão de literatura, que nos permite analisar publicações primárias e secundárias com diferentes tipos de delineamento sobre o tema de interesse, além favorece a identificação de lacunas de conhecimento para subsidiar a realização de novas pesquisas (BRUM CN, et al, 2015). Para 
nortear este estudo, formularam-se as seguintes questões: Em que fase da vida e por qual motivo a depressão e suicídio afeta os idosos? Quais as complicações desse quadro? O que pode ocorrer caso não haja um acompanhamento adequado?

Para isto, foram utilizadas algumas bases de dados online, sendo feita entre o final de 2019 a janeiro de 2020: 1) Literatura Latino-americana e do Caribe em Ciências da Saúde (LILACS); 2) Scientific Eletronic Library Online (SCIELO); e 3) Biblioteca Digital de Periódicos da Universidade Federal do Paraná (UFPR). Adotaram-se como critérios de inclusão: artigos científicos, idioma preferencialmente na língua portuguesa, títulos que adentrassem essa revisão de literatura e pesquisas relevantes dos últimos 10 anos. Já para os critérios de exclusão adotaram-se: dissertações e teses, resumos simples e expandidos, reportagens, textos duplicados, pesquisas com títulos e objetivos que fugissem da principal temática desta revisão e pesquisas publicadas a mais de 10 anos.

Das 119 pesquisas potencialmente relevantes, 58 pesquisas atenderam aos critérios do título e metodologia. Desses, 30 foram selecionadas para leitura íntegra e 28 foram excluídas após a leitura do resumo. Quando se aplicou o filtro das pesquisas mais relevantes dos últimos 10 anos, 5 foram excluídas por duplicidade e 15 por ano de publicação de mais de 10 anos. Restaram-se nove artigos que serviram de base para este estudo de revisão de literatura (Figura 1).

Figura 1 - Critérios de inclusão e exclusão.

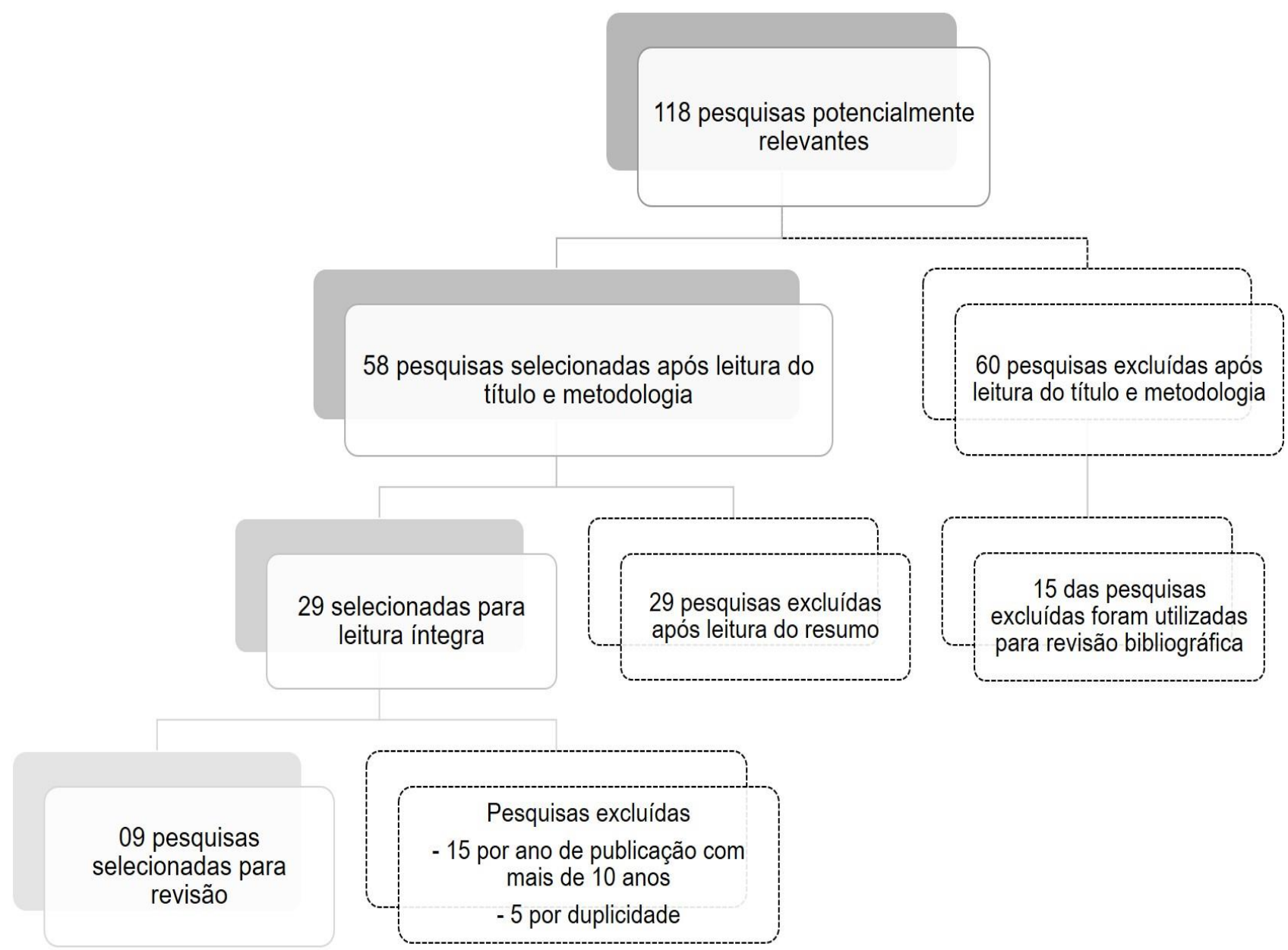

Fonte: Costa MAO e Cruz ACM, 2020.

Mediante os resultados de busca, foi realizada a seleção de todos os artigos encontrados. Os dados foram analisados com auxílio de uma planilha do Microsoft Excel®. E, em seguida apresentados em um quadro para melhor apresentação dos resultados da pesquisa. 


\section{RESULTADOS E DISCUSSÃO}

O suicídio como consequência da depressão é uma temática com vastas publicações realizada em diferentes locais e em diferentes idades acima dos 60 anos. Foi elaborada, portanto, um quadro de nove artigos selecionados para esta pesquisa com os seguintes parâmetros: (1) Nome do Artigo, (2) Autor(es), (3) Ano de publicação e (4) Objetivo (Quadro 1).

Quadro 1 - Caracterização dos artigos científicos segundo informações como: Nome do artigo, Autor(es), Ano de publicação e Objetivo. Manaus - AM, 2020.

\begin{tabular}{|c|c|c|c|}
\hline Nome do artigo & Autor(es) & Ano & Objetivo \\
\hline $\begin{array}{l}\text { Suicídio entre pessoas } \\
\text { idosas: revisão da literatura }\end{array}$ & $\begin{array}{l}\text { MINAYO MCS } \\
e \\
\text { CAVALCANTE } \\
\text { FG }\end{array}$ & 2010 & $\begin{array}{l}\text { Analisar fatores associados (doença } \\
\text { mental, enfermidades físicas e problemas } \\
\text { sociais) ao risco de suicídio de pessoas } \\
\text { idosas, a partir da literatura indexada nas } \\
\text { bases de dados nacionais e internacionais. }\end{array}$ \\
\hline $\begin{array}{l}\text { Autópsias psicológicas sobre } \\
\text { suicídio de idosos no Rio de } \\
\text { Janeiro }\end{array}$ & $\begin{array}{l}\text { MINAYO MCS, } \\
\text { et al. }\end{array}$ & 2012 & $\begin{array}{l}\text { Contextualizar e descrever o perfil dos } \\
\text { suicidas a partir de autopsias psicológicas } \\
\text { com base em entrevistas com familiares e } \\
\text { pessoas próximas. }\end{array}$ \\
\hline $\begin{array}{c}\text { Autópsias psicológicas e } \\
\text { psicossociais de idosos que } \\
\text { morreram por suicídio no } \\
\text { Brasil }\end{array}$ & $\begin{array}{l}\text { CAVALCANTE } \\
\text { FG e MINAYO } \\
\text { MCS }\end{array}$ & 2012 & $\begin{array}{l}\text { realizar no mínimo cinquenta autopsias } \\
\text { psicossociais com familiares de idosos que } \\
\text { haviam falecido por suicídio em dez } \\
\text { municípios das cinco regiões brasileiras }\end{array}$ \\
\hline $\begin{array}{l}\text { Caracterização das } \\
\text { tentativas de suicídio entre } \\
\text { idosos }\end{array}$ & $\begin{array}{l}\text { SELEGHIM } \\
\text { MR, et al. }\end{array}$ & 2012 & $\begin{array}{l}\text { Caracterizar as tentativas de suicídio entre } \\
\text { idosos e registradas de janeiro de } 2004 \text { a } \\
\text { dezembro de } 2010\end{array}$ \\
\hline $\begin{array}{l}\text { Diferentes faces da } \\
\text { depressão no suicídio em } \\
\text { idosos }\end{array}$ & $\begin{array}{l}\text { CAVALCANTE } \\
\text { FG et al. }\end{array}$ & 2013 & $\begin{array}{l}\text { Analisar diferentes faces da depressão } \\
\text { associadas a suicídio em idosos, a partir de } \\
\text { autópsias psicológicas }\end{array}$ \\
\hline $\begin{array}{l}\text { Conhecimento dos idosos } \\
\text { sobre os sinais e sintomas } \\
\text { da depressão }\end{array}$ & $\begin{array}{l}\text { ANDRADE } \\
\text { ABCA, et al. }\end{array}$ & 2016 & $\begin{array}{l}\text { Identificar o conhecimento dos idosos, } \\
\text { participantes do parque municipal do idoso } \\
\text { (Manaus - Brasil), sobre os sinais e } \\
\text { sintomas da depressão. }\end{array}$ \\
\hline $\begin{array}{l}\text { O comportamento suicida de } \\
\text { idosos institucionalizados: } \\
\text { histórias de vida }\end{array}$ & $\begin{array}{l}\text { MINAYO MCS, } \\
\text { et al. }\end{array}$ & 2017 & $\begin{array}{l}\text { Analisadas } 16 \text { histórias de vida de homens } \\
\text { e mulheres idosos que residem em } \\
\text { Instituições de Longa Permanência e que } \\
\text { tentaram dar cabo à vida ou têm } \\
\text { comportamento suicida. }\end{array}$ \\
\hline $\begin{array}{l}\text { Suicídio na Velhice - Um } \\
\text { estudo de Revisão } \\
\text { Integrativa da Literatura }\end{array}$ & $\begin{array}{l}\text { FERNANDES- } \\
\text { ELOI J e } \\
\text { LOURENÇO } \\
\text { JRC } \\
\end{array}$ & 2018 & $\begin{array}{l}\text { Revisão integrativa da literatura sobre } \\
\text { suicídio e velhice nas bases de dados } \\
\text { Scielo, Pepsic, LILACS e Index Psi no } \\
\text { período de } 2007 \text { a } 2017\end{array}$ \\
\hline $\begin{array}{l}\text { Suicídio entre idosos no } \\
\text { Brasil: uma revisão de } \\
\text { literatura dos últimos } 10 \\
\text { anos }\end{array}$ & $\begin{array}{l}\text { SANTOS } \\
\text { EDGM, et al. }\end{array}$ & 2019 & $\begin{array}{l}\text { Explanar sobre o suicídio na velhice no } \\
\text { Brasil por meio da revisão da literatura } \\
\text { existente. }\end{array}$ \\
\hline
\end{tabular}

Fonte: Costa MAO e Cruz ACM, 2020.

Os estudos de Minayo MCS e Cavalcante FG (2010) são os mais acessados e encontrados na literatura referente ao assunto em questão. Esses autores foram capazes de identificar as principais áreas de estudos que problematizam a assistência ao idoso, assim como os principais fatores que desencadeavam ou agravavam a enfermidade. Um dos principais pontos é associar questões de gênero e informações etárias para caracterizar grupos de risco, sendo esse um ponto diferencial dos outros trabalhos encontrados na literatura. Esses dois autores, em conjunto são responsáveis por descrever diversas nuances que envolvem a depressão e suicídio entre idosos. 
Dentre as pesquisas dos autores, uma abordagem diferenciada discute dados de autópsias psicológicas e psicossociais de idosos que morreram por suicídio no Brasil. Outra grande contribuição dos autores, é afirmação das narrativas que evidenciam a importância da presença dos profissionais do sistema único de saúde do Brasil - SUS no suporte às famílias, confirmando que a falta de atenção diferenciada à pessoa idosa gera os maiores riscos de suicídio (MINAYO MCS e CAVALCANTE FG, 2010).

Os resultados das pesquisas desses autores são valiosos para diversas áreas, e por meio de suas abordagens, pode-se compreender os diferentes fatores que contribuíram para prevenir, diagnosticar e realizar o tratamento de doenças mentais em idosos. Doenças estas que são atribuídas principalmente a depressão, ocasionada por questões de sofrimento físico e crônico à conflitos familiares. Outras causas encontradas são ocasionadas por fatores, como a perda de parentes próximos, aposentadoria mal planejada, dificuldade em aceitar o envelhecimento, câncer terminal, à eclosão de crises e à humilhação de internações psiquiátricas (MINAYO MCS e CAVALCANTE FG, 2010).

Os resultados dos autores também permitem concluir que as mulheres idosas têm mais ideação e produzem mais tentativas de suicídio, no entanto são os homens idosos os que mais concluem o ato de tirar a própria vida. Em contrapartida, dados publicados pelos mesmos autores no ano de 2012 indicam que homens e mulheres estão igualmente em risco quando constatado estado depressivo, e em ambos os grupos se encontravam idosos acometidos por doenças, deficiências e dores crônicas, reafirmando que outros fatores associados ao estado depressivo podem potencializar e estimular o ato suicida.

No entanto, reafirmam e caracterizam a principal causa para ambos os gêneros, concluindo que os homens são os mais afetados por estados depressivos e mulheres por conflitos familiares e conjugais, e em ambos os casos a depressão tem sido o principal fator associado ou não com outras variáveis que desencadeiam o ato (CAVALCANTE FG e MINAYO MCS, 2012).

Outro ponto importante para os profissionais da área de saúde e afins, pode ser encontrado nas pesquisas de Cavalcante FG e Minayo MCS (2012), os quais são responsáveis por identificar os grupos com maior risco de tentativa e execução do suicídio, sendo estes os idosos acima de 80 anos. Essa informação é de suma importância, uma vez que de forma prática, gestão de saúde e políticas públicas para a população idosa pode restringir o grupo de risco a atividades potenciadoras do não suicídio, gerando maior ressignificação a vida das pessoas na terceira idade.

Em publicações com autoria de Cavalcante FG, et al. (2013), os pesquisadores utilizam-se de métodos diferenciados, chamado de "método retrospectivo" para reconstitui as condições de saúde física e mental e as circunstâncias sociais das pessoas que se suicidaram a partir de entrevistas com familiares e pessoas com grau de afinidade às vítimas. O estudo, apesar de sua valiosa contribuição, possui amostra de dados muito baixa para que os resultados sejam generalizados, uma vez que, apenas incluiu pessoas de classe média e alta e de pessoas brancas.

Esse ponto é sabido entre os autores, visto que o autor em coautoria às publicações de Minayo MCS, et al. (2012) chamam atenção para a necessidade de atenção a diversidade da sociedade brasileira, migrantes e seus descendentes, índios e seus descendentes e dos pequenos povoados que também podem apresentar riscos que ocasionam situações semelhantes ao suicídio de idosos nas grandes cidades.

Apesar de baixa amostragem, o estudo de Cavalcante FG, et al. (2013) configura-se uma das principais publicações para área da saúde, uma vez que seus dados confirmam que há riscos de suicídio associado a mudanças na prescrição de medicamentos e/ou troca de médicos em fases críticas do tratamento do idoso. Esse resultado é uma importante informação e fonte de prevenção, uma vez que, ao diminuir o número de médicos que cuidam de pacientes idosos, também estaríamos prevenindo a saúde psicológica do idoso.

Os resultados da pesquisa também apontam que o suicídio em idosos é particular para cada pessoa e originado de diversas causas, pois cada um reage e enfrenta de modo específico. No entanto, a maioria das pistas de ajuda que os idosos deixaram para familiares e pessoas próximas, não foram levados a sério por parte deles (CAVALCANTE FG, et al., 2013). 
Outra narrativa dos autores e com uma baixa amostragem realizadas no ano de 2017 é constituída de informação valiosa para os profissionais que atuam na temática, esclarecendo percepções reais daqueles idosos que passaram ou passam por riscos de suicídio associados as tendências depressivas associadas com fatores de risco, podendo este não ser generalizado, mas utilizado com apoio e base para tomada de decisões no quesito prevenção. Neste estudo os autores puderam traçar percepções, sentimentos atitudes e práticas de um grupo de idosos com comportamento suicida (MINAYO MCS, et al, 2017).

As pesquisas de Minayo MCS e Cavalcante FG (2010), Minayo MCS, et al. (2012), Cavalcante FG (2013) e Minayo MCS, et al. (2017) são pesquisas complementares, com grande riqueza de informações que reafirmam e confirmam as causas que levam ao suicídio, sendo fontes de consultas para diversas áreas.

Outras abordagens como as de Seleghim MR, et al. (2012), corroboram com os estudos dos autores, em que o gênero com maior estatística de morte por suicídio são homens idosos e a depressão é o principal fator de risco.

A contribuição das pesquisas de Seleghim MR, et al. (2012), caracterizam as tentativas de suicídio entre idosos por meio de um estudo retrospectivo e documental do Centro de Informação e Assistência Toxicológica do Paraná (CIAT), demonstrando que idosos entre 60 a 69 anos tentaram suicídio com agrotóxicos, resultado este que contribui para prevenção de tentativas ao estabelecer, controle de usos dessas substâncias no cotidiano.

O estudo de Seleghim MR, et al. (2012) reforça a atuação da enfermagem na ocorrência destes eventos, sendo os profissionais os principais agentes chave para identificação e prevenção das causas mais comuns de suicídio na população idosa, os autores também apontam que mais estudos na área de enfermagem devem ser estimulados, pois há poucos dados epidemiológicos publicados, frente ao crescente índice de tentativas de suicídio entre pessoas idosas.

Um dos grandes questionamentos para os profissionais da área de saúde, é o questionamento do papel do estado em relação a essa problemática, por esse motivo, os estudos de Andrade ABCA, et al. (2016) realizada no estado do Amazonas, preenche parcialmente a lacuna e complementa as abordagens dos autores neste estudo, em virtude de contribuir com a caracterização do perfil sociodemográfico que influência a saúde mental do idoso, gerando fatores de risco para a depressão.

Os autores enfatizam ainda, a importância dos familiares e os profissionais de saúde, assim como os outros estudos discutidos neste trabalho, demonstrando que a assistência e acompanhamento familiar é importante para prevenção da saúde psicológica do idoso.

Os resultados de Andrade ABCA, et al. (2016) identificaram a falha no sistema de saúde, principalmente em relação ao diagnóstico dos sintomas depressivos e todos os aspectos que envolvem a causa. Essas informações são importantes do ponto de vista prático, pois servem de base e apoio informacional para mudanças no sistema de saúde pública, em especial as que tratam da saúde e prevenção ao suicídio na terceira idade

Por meio dos estudos anteriormente citados, o de Fernandes-Eloi J e Lourenço JRC (2018) é um dos mais recentes, e os resultados corroboram com complementaridade os diversos resultados anteriormente discutidos. A abordagem dos autores esteve restrita a três categorias analíticas, sendo estas: fatores de risco, relações familiares/suicídio na velhice e saúde pública.

Artigos cuja temática abrangia a categoria "fatores de risco" puderam identificar diversos riscos associados ao comportamento suicida em idosos, no entanto a depressão é o fator que mais contribui, visto que desencadeia uma série de outros problemas psicológicos, como por exemplo, falta de concentração.

Quando o suicídio está relacionado a doenças crônicas, as consequências incapacitantes destas tem sido o principal fator que desencadeia 0 ato, assim os autores reafirmam a necessidade de ressignificação da velhice para que durante a chamada "melhor idade" os idosos não vivenciem estádios depressivos e suas consequências. Esses resultados corroboram os achados de Minayo MCS e Cavalcante FG (2010), Minayo MCS, et al. (2012), Seleghim MR, et al. (2012), Cavalcante FG (2013), e Minayo MCS, et al. (2017). 
$\mathrm{Na}$ categoria "relações familiares" os artigos discutidos apontaram que relações familiares fragilizadas associadas com negligências, abusos e violência contra o idosos são os principais fatores que consumam o ato suicida na terceira idade.

Esses abusos podem aparecer de forma física, psicológica, financeira e/ou sexual, concluindo que a família infelizmente não é sinônimo de segurança e proteção para o idoso, pois o desamparo tem sido a principal problemática que induz o sofrimento e desencadeia ideações suicidas nos idosos (FERNANDESELOI J e LOURENÇO JRC, 2018).

Por fim, na categoria "saúde pública e suicídio na velhice" os autores discutidos apontam que, em um contexto nacional há uma ausência de políticas públicas efetivas e discrepâncias em relação a ações preventivas continuadas.

Fernandes-Eloi J e Lourenço JRC (2018), enfatizam no trabalho que o suicídio em idosos é um grave problema de saúde pública e a gestão de saúde fragilizada quando se trata do assunto suicídio em idosos não contribui e nem minimiza os números de suicídios na terceira idade, pelo contrário, tem aumentado significativamente os dados e expectativas de vida.

Esses resultados reafirmam os resultados de Seleghim MR, et al. (2012), realizados no estado do Amazonas, destacando as falhas que o estado comete ao tratar problemas psicológicos da sociedade, desamparando comunidades mais fragilizadas por meio de atendimentos com escassez de remédios, médicos, amparo, cordialidade nos serviços e compromisso com os pacientes.

Os dados reunidos por meio do artigo dos autores Fernandes-Eloi J e Lourenço JRC (2018) é uma valiosa contribuição para literatura, atende as expectativas para o entendimento do atual cenário que envolve a problemática e contribui para novas investigações sobre a realidade atual dos idosos possibilitando novas perspectivas sobre envelhecimento com saúde e qualidade de vida da população idosa.

Pesquisas recentes realizadas por Santos EDGM, et al. (2019), sobre suicídio entre idosos no Brasil, trata de uma revisão de literatura dos últimos 10 anos. A maior contribuição da abordagem é explanada nas considerações, revelando que ainda nos atuais tempos não há uma literatura que contribua para maior embasamento e conhecimento de todos os aspectos que envolvem o suicídio na população idosa, porém sugerem que estudos que abordem a influência midiática, geografia do suicídio de idosos no país, fatores preceptores do suicídio como forma de antecipação da morte são assuntos importantes que devem ser trabalhados nas áreas de interesse, uma vez que essas informações podem ser usadas para novas formas de abordagem e prevenção da população idosa, principalmente idosos na faixa etária de risco (SANTOS EDGM, et al., 2019).

O estudo confirma o que as pesquisas anteriores têm reafirmado, sendo eles, que os homens idosos possuem mais casos registrados de consumação do ato, enquanto as mulheres apresentam-se nas estatísticas de tentativas.

Também é encontrado, assim como nos outros estudos que o serviço público não consegue atender e precisa se reestruturar para tratar de questões como o suicídio na terceira idade. Ressalta-se ainda a importância da família como os principais agentes de atenuação ou diminuição das tentativas de suicídio, sendo um dos principais aspectos trabalhados em conjunto para prevenção de depressão e tendências suicidas nos idosos da família (SANTOS EDGM, et al., 2019).

\section{CONSIDERAÇÕES FINAIS}

O estudo verificou que os principais fatores de risco relacionados ao suicido em idosos é a depressão, ocasionada pela aposentadoria, problemas de relacionamento familiar, perda de habilidades, diminuição de possibilidade de escolhas. Ressaltam-se as diferenças de gênero que diversos autores analisaram. As mulheres idosas têm mais ideação e produzem mais tentativas de suicídio, no entanto são os homens idosos os que mais concluem o ato de tirar a própria vida. Os resultados desta revisão evidenciam as associações de diversos fatores encontrados na literatura com o suicídio, também aponta meios para promover a saúde física e mental em idosos diminuindo, assim, os riscos de suicídio entre essa população. 


\section{REFERÊNCIAS}

1. ANDRADE ABCA, et al. Conhecimento dos idosos sobre os sinais e sintomas da depressão. Saúde em Redes, 2016; 2(2): 157-166.

2. BARRETO J. Tratamento actual da depressão no idoso. In: FIRMINO H, et al. (org.). Psicogeriatria. 1nd ed. Coimbra: Psiquiatria Clínica, 2006.

3. BATISTA AS, et al. 2008. In: Envelhecimento e Dependência: Desafios para a Organização da Proteção Social. Brasília: MPS, SPPS.

4. BRASIL. Secretaria de Direitos Humanos da Presidência da República. Manual de enfrentamento à violência contra a pessoa idosa. 2014. 90 p.

5. BRUM CN, et al., 2015. Revisão Narrativa de literatura: aspectos conceituais e metodológicos na construção do conhecimento da enfermagem. In: LACERDA MR, COSTENARO, RGS (org.). Metodologias da pesquisa para a enfermagem e saúde: da teoria à prática. 1nd ed. Porto Alegre: Moriá, 2015.

6. CARREIRA L, et al. Prevalência de depressão em idosos institucionalizados. Revista Enfermagem UERJ, 2011; 19(2): 268-273.

7. CAVALCANTE FG, MINAYO MCS. Autópsias psicológicas e psicossociais de idosos que morreram por suicídio no Brasil. Ciência \& Saúde Coletiva, 2012, 17(8): 1943-1954.

8. CAVALCANTE FG, et al. Diferentes faces da depressão no suicídio em idosos. Ciência \& Saúde Coletiva, 2013; 18(10): 2985-2994.

9. CRUZ DT, et al. Envelhecimento populacional e bases legais da atenção à saúde do idoso. Cadernos de Saúde Coletiva, 2010; 18(4): 500-508.

10. FERNANDES-ELOI JE, LOURENÇO JRC. Suicídio na Velhice - Um Estudo de Revisão Integrativa da Literatura. Rev. CES Psico, 2019; 12(1): 80-95.

11. MEDEIROS JML. Depressão no idoso. Dissertação (Mestrado Integrado em Medicina) - Faculdade de Medicina, Universidade do Porto, Porto, 2010; $31 \mathrm{p}$.

12. MINAYO MCS, CAVALCANTE FG. Suicídio entre pessoas idosas: revisão da literatura. Revista de Saúde Pública, 2010; 44(4): 750- 757.

13. MINAYO MCS, et al. Autópsias psicológicas sobre suicídio de idosos no Rio de Janeiro. Ciência \& Saúde Coletiva, $2012 ; 17(10): 2773-2781$.

14. MINAYO MCS, et al. O comportamento suicida de idosos institucionalizados: histórias de vida. Physis Revista de Saúde Coletiva, 2017; 27(4): 981-1002.

15. SAMPAIO LS, et al. Condições sociodemográficas e de saúde de idosos residentes em domicílio no município de Jequié - BA. Revista Brasileira de Geriatria e Gerontologia, 2009; 12(2): 267-274.

16. SANTOS EDGM, et al. O. Suicídio entre idosos no Brasil: uma revisão de literatura dos últimos 10 anos. Psicología, Conocimiento y Sociedad, 2019; 9(1): 258-282.

17. SANTOS EGO, et al. Análise espaço-temporal da mortalidade por suicídio em idosos no Brasil. Rev. Bras. Geriatr. Gerontol., 2017; 20(6): 854-865.

18. SELEGUIM MR, et al. Caracterização das tentativas de suicídio entre idosos. Cogitare Enferm., 2012; 17(2): $277-283$.

19. SOUSA L, RIBEIRO AP. Prestar cuidados de enfermagem a pessoas idosas: experiências e impactos. Saude soc, 2013; 22(3): 866-877.

20. TEIXEIRA LMF. Solidão, depressão e qualidade de vida em idosos: um estudo avaliativo exploratório e implementação-piloto de um programa de intervenção. Dissertação (Mestrado Integrado em Psicologia) - Faculdade de Psicologia, Universidade de Lisboa, Lisboa, 2010; 101 p.

21. VALCARENGHI RV. Alterações na funcionalidade/cognição e depressão em idosos institucionalizados que sofreram quedas. Acta Paulista de Enfermagem, 2011; 24(6): 828-33. 